\title{
FairPeers: Efficient Profit Sharing in Fair Peer-to-Peer Market Places
}

\author{
Giancarlo Ruffo · Rossano Schifanella
}

Published online: 21 June 2007

(C) Springer Science+Business Media, LLC 2007

\begin{abstract}
The technical impact of the Peer-to-Peer (P2P) paradigm on content distribution applications has been proved successful and efficient, when participants cooperation is achieved. Conversely, the business model is not clear: given a copyprotected object, its owner must be paid back for each transaction taking place from a provider to a receiver. The $\mathrm{P} 2 \mathrm{P}$ paradigm assumes that a receiver turns into a provider, but it is questionable why she/he should provide properly the content, if the owner wants to be reimbursed. Actual systems introduce fairness, giving incentives (e.g., a differential service, like in BitTorrent) to altruistic peers, with the consequence that the owner of an object is economically damaged everyday. Hence, music and film industry sees P2P techniques as a hostile framework for distributing copy protected content for free: today's answer of the industry is investing in DRMbased solutions, that are not interoperable between different devices and players. In this paper, we present FairPeers, a P2P market framework, that joins a straightforward intellectual property protection and a fair economic model by maintaining the efficiency typical of P2P file sharing systems. The study is completed with an exhaustive security analysis, and the description of a prototype implementation that shows that the $\mathrm{P} 2 \mathrm{P}$ paradigm is mature enough to present to the broadest community new revenue models, simply using available tools and state-of-the-art techniques.
\end{abstract}

Keywords Distributed applications · Accounting and payment · Copyright protection · Incentive-based management

\section{Introduction and Scope}

Popular Peer-to-Peer (P2P) systems have gained a bad reputation through file sharing applications, despite their technical advantages, because they make easy for

G. Ruffo $(\bowtie) \cdot$ R. Schifanella

Computer Science Department, University of Turin, Corso Svizzera 185, 10149 Turin, Italy

e-mail: ruffo@di.unito.it 
the users to access copy-protected files for free. Amongst the many potentially damaged actors, the music and film industry (represented mainly by Recording Industry Association of America (RIAA) and Motion Picture Association of America (MPAA)) fiercely targeted P2P applications during their campaign against piracy. Even if Internet users are quite upset for this reaction, and they complain that their privacy and freedom of speech are under attack, it goes without saying that copyrights are violated everyday and, since it is very difficult to trace the peers' activity, identification of abuses cannot be fairly performed because of the absence of a central authority.

In addition, a $\mathrm{P} 2 \mathrm{P}$ revenue model is hard to find. Let us adopt the terminology used by Hummel, Muhle, and Schoder in [1], assuming that a payment takes place when a copy-protected digital file is downloaded: given a transaction object (or simply an object), we need to discriminate between the owner and the provider(s) of the object: in fact, in a P2P system the provider can upload the object to the receiver without the rights of being accountable for providing the given copy-protected content. From this point of view, the question is if other rational agents than the owner should be involved in such a transaction. Moreover, the receiver of the bought object can become the distributor later on, but why should he/she provide properly the content if the owner wants to be reimbursed? Furthermore, since the choice of collaboration could give rise to a significant communication and computational burden $[2,3]$, the users might choose not to cooperate pursuing the self-interest first. This problem is just the other side of the coin of the well known free-riding [4] phenomenon, and, as we will briefly overview in section background and related work, it is dealt with incentives.

In order to improve the understanding of the biggest picture, let us recall the recent Steve Jobs' “Thoughts on Music' [5], pointing out the Digital Rights Management (DRM) systems that Apple has resolved to adopt for protecting its music against piracy. The reasons for such a "riot'" are the following: DRMprotected digital music is prevented from being played by devices of different producers; conversely, DRM-free content, that uses "open" formats (e.g., MP3 for music and MPEG4 for movies), can be downloaded, distributed, copied and played on different devices. This is an implicit disincentive to legally buy copy-protected digital content, because DRM-free files are interoperable: in fact, $97 \%$ of the music filling iPods is unprotected and of obscure origins. Jobs' conclusions are quite astonishing: abolishing DRMs entirely and selling music encoded in open licensable formats. However, there is no obvious reason for believing that piracy would decrease even if the Steve Jobs' dream for a "DRM-free", world will finally occur. This implies that future legal market models have to consider serious, scalable, efficient, secure and reliable alternatives to DRM-based on-line (centralized) stores. The Peer-to-Peer paradigm provides a quite mature framework for this applicative domain, making digital content sharing applications a valid solution even for small vendors and emerging artists. In fact, small-medium parties of a market place could hardly afford production and maintenance costs that can be very high if distribution is provided by means of a resilient client-server architecture (e.g., iTunes, Yahoo!, Microsoft Media Shop). Finally, social networking and self organized communities can be exploited in order to enforce epidemic phenomena and word of mouth 
marketing [6], and-again - the $\mathrm{P} 2 \mathrm{P}$ paradigm is in principle the most scalable platform for running highly distributed services.

FairPeers, the market-place framework presented in this paper, gives a solution to these problems: it proposes a DRM-free P2P content distribution platform, in combination with a license-based copyright protection mechanism and a revenue model that gives incentives to providers as well as to owners. After an overview concerning related works in section background and related work, the service model is described in section service model. The protocol defined in section FairPeers: a fair market for profit sharing addresses the solutions to technical problems that arise when dealing with such a complex domain. In particular, security aspects are analyzed in detail, and economic transactions have been "built-in" the distribution framework, in order to maintain the distinctive efficiency of the P2P paradigm: the "multi-source downloads" option has been preserved, as it is well known that it dramatically increases transfer rates. Security considerations are addressed in section security analysis and reputation management, and intuitions that motivated our study are supported by experimental results in a simulative environment described in section simulations. Finally, an overview of the peer's structure and present prototype implementation is given in section peer's architecture and implementation.

\section{Background and Related Work}

Selfish behavior of users is at the basis of the spread of free-riding phenomena. A free-rider might be defined as a peer that takes advantage of the system, exploiting the effort of other peers, e.g. searching for files or downloading liked contents, without any contribution in terms of performed tasks or shared resources.

The intrinsic characteristics of the $\mathrm{P} 2 \mathrm{P}$ model that fosters the spread of a freerider behavior are considerably: highly dynamic memberships, the presence of cheap identities, even called pseudonyms, the untraceability of the users' actions and the possible collusions of malicious nodes.

Several empirical studies have been performed concerning the evidence of the free riding in real file sharing communities [4, 7, 8]. For example, after monitoring samples of messages on Gnutella network during a day of year 2000, Adar and Huberman observed that nearly $70 \%$ of Gnutella users share no files, and nearly $50 \%$ of all responses are returned by the top $1 \%$ of sharing hosts [4].

In general, a peer can behave as a free rider in different situations: (1) not sharing any resource but only profit by system's features, or (2) not cooperating in the standard interaction protocol or not providing the basic functionalities. The first case is the most common behavior in file sharing environment where the majority of users does not share files but only downloads them. Instead, in the second scenario, a peer can decide not to forward the searching requests he receives or, in a generic distributed hash table system, he can maliciously not provide the content that he is responsible for. Anyhow, the peer is inherently unreliable, due to faults in communication links or as a result of a system crash or simply due to the high turnover rate typical of peer-to-peer networks. Unluckily, it is generally impossible 
to distinguish if a peer acts maliciously or it is only unreliable. It is a general opinion that redundancy [9] is the only valid solution to counter this effect.

A straightforward solution is to rely on peers' altruism: empirical studies in behavioral economics argue that merely self-interested based approaches usually fail to explain the observed behavior of people [10]. Moreover, Andreoni [11, 12] noted that altruistic actions may be part of the rational behavior of a human individual: when people make decisions about giving, they are influenced by many factors such as social pressure, sympathy, guilt, desire to earn prestige, friendship or respect. According to these premises, Feldman et al. remarked that if the degree of social generosity is sufficiently high, no intervention is necessary to counter the free-riding phenomenon [13]. Anyhow, in a Peer-to-Peer environment such altruistic hypothesis are not sufficient to counter the predominant egocentric behavior of users observable in modern file-sharing communities or in other distributed applications. In fact, there are some relevant differences on respect to social context: feelings such as sympathy, friendship or longing of prestige are not present in an environment characterized by anonymity between peers, lack of accountability of resource consumption and actions, and absence of permanent identities. It goes without saying that in a market place where receivers pay for accessing a service, all these issues are very relevant and critical.

It is necessary to introduce some mechanisms that provide incentives and stimulate cooperative behaviour between users in order to fight the negative outcomes of free-riding, like degradation of the performance, unpredictable and fitful availability of resources, or, under the most unfavourable conditions, the complete collapse of the system.

In order to design effective incentives to cooperate, two basic categories of solutions have been proposed:

- Trust-based incentives: the interaction between peers is stimulated by the trust that one participant puts in the other parties. Reputation mechanisms belong to this category. In other words, in order to benefit of services or resources in the systems, a peer needs to maintain a good reputation by means of a cooperative and honest behavior.

- Trade-based incentives: in respect to the trust-based approaches, the trade based mechanisms explicitly remunerate a cooperating peer in terms of a service in return. The receiver can recompense the provider immediately, during or after the service provision, or she can promise a service in return. In the first case, we obtain barter trade patterns; instead, the second case, we talk of bond based patterns. Afterward, we will give a more detailed overview regarding these two approaches:

- Barter-based: accordingly with the meaning of a barter in the real life, this scheme is characterized by the ability of the consumer to remunerate the provider by simultaneously offering a service in return. It is clear that both the participants are constrained to benefit of the service simultaneously offered by another party. This approach guarantees anonymity between peers and it offers a better scalability in relation to the trust-based schemes, but it suffers from a very poor flexibility. In last analysis, the barter-based scheme 
has been successfully applied in file sharing systems: the tit-for-tat strategy in BitTorrent [14, 15] and the credits system in Emule [16] are two well known examples.

- Bond-based: this scheme specifies that the consumer transfers to the provider a bond that promises a service in return. A main characteristic of bonds is that they can be transferred. The transferability of the bonds are a suitable property because the provider can spend collected promises in future cooperations. Nevertheless, the introduction of a form of money insinuates critical problems like forgery and double spending. Another problem raised by bond-based schemes is the user's acceptance [17, 18]. In fact, several economic studies have sharpened as the flat-fee economic model can generate greater profits per good on respect to the pay-per-use model depicted in monetary systems [19, 20]. Examples of bond-based mechanisms implemented in popular file sharing systems are the digital currency named Mojo in MojoNation [21] (actually the original commercial project is closed) and the Alnet's points in KaZaA [22].

Also Game Theory [23] has been extensively used to analyze the behavior of peers and the free riding phenomenon. Game theory considers peers like rational and autonomous agents, namely also players, guided by an utility function that depicts their preferences regarding the different outcomes of the game.

Several proposals based on such domain model have been introduced [24-30].

Furthermore, other approaches to build effective incentives schemes are based on the Mechanism Design (MD) and Distributed Algorithmic Mechanism Design (DAMD) [31] concepts. MD is a sub-field of the game theory that investigates the design of rules to achieve a specific outcome in a game [31-33]. The relevance of these studies shows that every kind of Peer-to-Peer applications have to deal with incentive mechanism to gain success.

Since in a market domain incentives could achieve a value in terms of revenues, the peculiarity of the $\mathrm{P} 2 \mathrm{P}$ paradigm must be carefully analyzed. An interesting scenario's description comes from the Market Management of Peer-to-Peer Services (MMAPPS) research project, that designed, built and tested a middleware able to provide management support for Peer-to-Peer services, exploring new charging options and business models. After the identification of a set of functional and nonfunctional requirements, the project introduced the basic architectural concepts and, in particular the features of three models, namely the market, service and peer models, that are the basis of the Service Oriented Peer-to-Peer Service (SOPPS) [34] architecture.

Basically, the service model outlines the different types of services a peer can offer to other peers and the interfaces through they can be accessed. The market model provides the market-oriented view of a Peer-to-Peer system, identifying the roles and the dynamics by means of peers that buy and sell services. Finally, the peer model depicts the internal structure of a peer along with the functionalities needed to provide services and to endorse the market (for a detailed survey see [35]). 


\section{Service Model}

Our service model shows a structure that can be logically divided in three different levels: (1) the overlay level, (2) the accounting level, and (3) the market level. These modules give different services to other modules, and even if we can think at them, at this present degree of abstraction, as a pile of protocols, applications can use functions and operations offered at different levels. Lets define each module in terms of the functions they export to the other parts of the framework (see Fig. 1):

Overlay Level

The overlay network can be unstructured or structured. Gnutella [36], and Kazaa [22] are examples of notorious unstructured overlays; Pastry [37], Chord [38] and Kademlia [39] are instances of structured networks. Systems in the second group of overlays are considered more scalable than the others. Very roughly, structured overlays are defined in terms of a topology (i.e., a forest, a ring, and so on), a routing mechanism, and an identifier space, which is used to uniquely locate nodes and resources in the network. The key idea is that each node $\mathrm{N}$ is responsible for a set of resources whose identifiers are 'closer' to N's identifier than to the others; in fact, a distance metric is defined for each different system.

Usually, a distributed storage service is defined over this basic layer. It is possible to insert a new resource in the network, that will be assigned to the node of responsibility. Hence, given a generic node $\mathrm{N}$ and a resource $\mathrm{R}$, respectively identified by $\mathrm{id}_{\mathrm{N}}$ and $\mathrm{id}_{\mathrm{R}}$, the overlay layer should be able to export the following functions:

- $\quad \operatorname{route}\left(m, i d_{N}\right)$ : it routes message $m$ to node $\mathrm{N}$;

- $\operatorname{search}\left(i d_{l}\right)$ : it looks for 1 , and returns a pointer to the node (or the set of nodes) that is responsible (or that caches) the searched resource.

- $\operatorname{insert}(l)$ and delete $(l)$ : these functions are used to store and to remove a given resource to a node. Some systems implement basic authentication mechanisms:

Fig. 1 Logical levels of the service model

\section{Market Level

\begin{tabular}{|c|c|c|c|}
\hline Rights & Management & Trust & Reputation \\
\hline Pricing & Auctioning & Fairness & Content Distribution \\
\hline
\end{tabular}

Accounting Level

$\operatorname{pay}\left(\mathrm{id}_{\mathrm{x}}, \mathrm{id}_{\mathrm{\gamma}}, \mathrm{v}\right)$

Overlay Level

$\operatorname{route}\left(m, i_{N}\right) \quad \operatorname{search}\left(i_{1}\right)$ insert(I) delete(l) 
for example, only a node with given credentials can remove a resource from the network.

Observe we do not include "retrieve" or "download" operations at the overlay level, that is only able to locate a given resource (with search $\left(\mathrm{id}_{l}\right)$ ).

Finally, consider that such operations can be managed in a centralized manner. An example is Napster [40], even if the most famous market place on the Internet that implements centrally these functions is eBay.

\section{Accounting Level}

In order for a market to take place, security concerns at this level should be considered very seriously. We have to manage both macro-payment and micropayment transactions; frauds are common; services and resources consumption must be accounted as well. The most part of actual markets adopt a central authority that manage economic transactions (e.g., PayPal) when services are furnished or items are sold. At the resource level, many credit-based incentive mechanisms have been proposed over the last years. However, the accounting level must provide functions for crediting or debiting users (with currency with or without legal value). For the sake of simplicity, we define only the following function:

- $\operatorname{pay}\left(i d_{X}, i d_{Y}, v\right)$ : it invokes all the measures in order to securely provide a payment of value $\mathrm{v}$ from user $\mathrm{X}$ to user $\mathrm{Y}$.

The pay method can be implemented by way of a central authority (very common, and maybe preferable, for managing macro-payments) or of a distributed system. In the latter class of proposals, many strategies can be further classified as Local Accounting, Token-Based Accounting, and Remote Accounting (e.g., see [1]).

\section{Market Level}

This level includes most of the services as perceived by the final user. We list here a set of properties and functions that a framework could implement, and that are tightly coupled to the market model.

- Pricing: The pricing function maps a service onto a tariff function or a scalar value that basically represents the price of the service consumption. It can be fixed or competitive; in the second case, each provider of the same service can serve it at different prices. For example, in a storage application, some peer can offer slices of its own disk space at a lower price than a competitor.

- Auctioning: When the good under sale is in limited number, different bids can be taken into consideration by the merchant. In virtual markets that deal only with electronic sources (different replica can be easily produced), auctioning is less important.

- Copyright Management: As it is stated in the introduction, one of the most relevant motivation to our study is understanding how a proper copyright management can be included in a proposal without mitigating the technical benefits of a P2P system. In general, such a topic is critical in existent digital 
marketplaces and on line stores, and one can simply ignoring the problem, introducing a DRM compliant system (e.g., iTunes, FairPlay or Napster to Go) or selling DRM-free content (Yahoo! And Amazon recently announced this option). Observe that, whenever possible, the responsibility and actions accountability are transferred to the final users (e.g., eBay, PeerMart).

- Fairness: If the owner is paid, then also the provider, that contributes to the system with his own bandwidth, cpu cycles, and disk space, should be (at least partially) paid back. We think that this property has been deeply underestimated and a proper implementation of fair mechanisms can strongly incentive users to behave legally and can bring the success to a market place.

- Content Distribution: No electronic item must be delivered by traditional shipping (e.g., air mail) methods. On the contrary, electronic content can be distributed depending on the kind of service. In fact, delay-tolerant (DT) connections (e.g., TCP based) can be used for file sharing applications, but timesensitive (TS) mechanisms are needed when audio/video (e.g., a soccer match) is streamed to a set of paying users.

- Trust and Reputation: When a transaction is completed (or even maliciously aborted), involved participants can be asked to submit a feedback, that can be negative or positive. We will spend some other words about the importance of reputation management in section conclusions and future works. Reputation management is one of the most intriguing proposals against free riding.

In Table 1 we compare different market places and on line stores (most of them operative). It is possible to observe that FairPeers is the only Peer-to-Peer proposal supporting both Copyright Management and Fairness, adopting a license based DRM-free solution (see section FairPeers: a fair market for profit sharing). Moreover, even if the actual implementation of FairPeers provides only delay tolerant content transmission (and a file sharing service), the "lottery" extension of this system (see section security analysis and reputation management) can be exploited for streaming audio/video in a CoolStreaming-like [41] fashion.

Table 1 Comparison of different market places on line stores (n.a. is for "not applicable')

\begin{tabular}{llllllll}
\hline & Pricing & Auctioning & \multicolumn{2}{l}{ Copyright protection } & Fairness & Distribution & Reputation \\
\cline { 3 - 6 } & & & DRM & DRM-Free & & & \\
\hline Amazon & Fixed & No & Yes & Not Yet & n.a. & Mixed & n.a. \\
I-Tunes & Fixed & No & Yes & Not Yet & n.a. & DT & n.a. \\
eBay & Competitive & Yes & No & No & n.a. & Mixed & Yes \\
MojoNation & Fixed & No & No & No & No & DT & No \\
Napster & Fixed & No & Yes & No & No & DT & No \\
PeerMart & Competitive & Yes & No & No & n.a. & n.a. & No \\
FairPeers & Fixed & No & No & Yes & Yes & DT/TS & Not Yet \\
\hline
\end{tabular}




\section{FairPeers: a fair market for profit sharing}

An earlier version of FairPeers has been proposed in [42, 43], as a protocol overlaying PPay, including a peculiar and fair copyright management feature: when a file download occurs, providers are paid back for giving the service, as well as the owner. In this section, we add two innovations: first of all, we present FairPeers unbound from PPay. In fact, other payment mechanisms can be used as well, in order to maintain the reference model discussed in section service model. It is well known that such a modularization allows nice properties and eases updating of the system. In particular, even if in the actual prototype (see section peer's architecture and implementation) we use PPay as the main payment management module, we want that future changes of implementation of that layer's service remain transparent to the rest of the framework. For example, distributed accounting (e.g., PeerMint [44]) could be used instead of token based accounting, adding scalability to the entire system.

Another, and most relevant, difference with the previous version of FairPeers, is that it can deal with multiple source downloads: the old framework was limited to one-to-one transactions, that is a quite unrealistic scenario. The Rivest's Electronic Lottery Tickets scheme [45] has been of inspiration for us during the design of this extension, and such a proposal is really useful to improve efficiency and scalability to the system, and to significantly reduce selfish behaviors, because most people would like to participate to a captivating challenge. A complete analysis of the multi-source downloads option and the related lottery scheme is given in section purchasing an object from many providers

In order to model the domain we introduce the following actors:

- A copy-protected Object f(e.g., a song, a movie, an executable program,...).

- The Mediators that facilitate the interaction between the actors of the market and provide some basic functionalities. There are several forms of mediating services with respect to the different modules:

- The Copyright Grantor (CG) that stores the ownership's information of all the objects inserted in the system. It may also certify the cost of an object as decided by the owner, as well as other information about the content (e.g., name of the band, genre, production year,...).

- The Certification Authority (CA) that certifies users' identities. FairPeers assumes the presence of a Public Key Infrastructure (PKI) providing each party with digital certificates that validate their authenticity. Accordingly, each peer is furnished with a private and a public key guaranteed by the CA.

- Several other mediators can be introduced as well. For instance, in a scenario where the accounting service is implemented by way of the PPay protocol (as our current prototype does), the presence of a Broker that mints and cashes the coins or that discovers frauds is mandatory. In general, the presence of a Trusted Third Party could facilitate the interactions between users, i.e. in the case of conflicts resolution.

- The owner of an object $f$, that deposits to the Copyright Grantor all the information about $f$. It can be the author of $f$, or who owns the copyright of $f$. She 
can also proclaim a set of peers that could act as owner's delegates, and that can act on behalf of the real owner.

- The copyright certificate is issued (and signed) by the grantor, and is an unforgeable message that binds $f$ with an owner.

- The distribution license is issued by the owner (of by one of her delegates), when she authorizes a peer to further distribute a given content.

- The provider is a regular peer that has gained the distribution license for a given $f$. Usually, a peer is authorized to distribute $f$ immediately after he received it.

- The receiver is a peer that buys and downloads an object $f$ directly from the owner or via a licensed provider.

Notice the difference between an owner's delegate and a provider: the first is accountable for $f$, and can cash a given payment on behalf of the owner. Moreover, an owner's delegate is authorized to show the copyright certificate and to issue other distribution licenses. A provider can only upload (part of) the file he has been authorized to distribute, and can be accounted only for service sharing. Of course an owner's delegate can be also a provider.

It is worth underlining that the business model has to take carefully into account the fundamental role played by the mediators. For instance, the CG is needful to guarantee the binding between an object and the related owner and, without it, the system stops working. A similar observation can be made for the CA that assures the security level of the underlying PKI. The Broker in a PPay-enabled market is another prominent example. As well as all the participants in the trading process, e.g. the owner, the provider and the receiver, have to be refunded, the revenue model has to consider and, therefore, remunerate the leading role of mediators. A comprehensive analysis about the most suitable approach to account mediators' services is out of the scope of this proposal and requires careful economic considerations. In any case, a feasible solution could be to set a fixed fee that each user has to pay when he joins the system. This overall revenue can be distributed amongst the mediators, accordingly to their involvement (e.g., in proportion to the managed transactions).

Someone could observe that the presence of mediators reduces the "pureness" of the P2P paradigm and that can present single points-of-failure. However, under the users' point of view, the presence of authoritative third parties makes authentication functionalities even more trustable [46]. In addition, bottlenecks can be significantly reduced if the $\mathrm{P} 2 \mathrm{P}$ framework is able to absorb the largest amount of transactions.

\section{The Copyright Certificate}

The Copyright Grantor furnishes a simple accounting service, and even if it can be implemented as a pure distributed system, for simplicity we prefer to think of it as an external and central trusted third party. When an owner $A$ wishes to sell a digital content he created, he starts a session with the CG, by submitting the file he wants to sell in the $\mathrm{P} 2 \mathrm{P}$ market place. Hence, owner $A$ sends following certification request to the $\mathrm{CG}$ : 


$$
f,\left\{i d_{f}, i d_{A}, \bar{f}, v, D L\right\} \operatorname{Sig}(A) .
$$

where $i d_{f}$ and $i d_{A}$ are respectively unique identifiers of $f$ and $A$. They can be Pastry identifiers, or any fixed length bit sequence returned by a collision resistant hash function. For example, $i d_{f}=\mathrm{SHA}-1(f)$ and $i d_{A}=\mathrm{SHA}-1(\operatorname{PK}(A))$, where $\mathrm{PK}(A)$ is the public key of user $A$. Furthermore, $\bar{f}$ contains some meta-data (e.g., genre, coowners, session artists, production year) about $f$, that can be used for verification purposes, or also for matching search criteria during look up. Finally $v$ is the value that the owners assigns to $f$, and $D L$ is the delegates list, made of a sequence of peers' identifiers. Note that this certificate admits null values, i.e., $v=0$, and $D L=\emptyset$. The request is signed with $\operatorname{Sig}(A)$ : in fact, such message should be not repudiable by $A$, because in the case of a dispute (e.g., plagiarism), CG should provide reliable information to a third party.

In our model we assume that the CG does not bear any responsibility about the submitted content. More precisely the CG checks whether or not the given object was already submitted by someone else, but, in case of disputes, only the user is required to prove the originality of the object. Next, CG creates the following copyright certificate and sends it to $A$, that will store it for future use:

$$
\operatorname{Cert}(f)=\left\{i d_{f}, i d_{A}, f, L S, v, D L\right\} \operatorname{Sig}(C G)
$$

where $L S$ is the life span of the given certificate. Moreover, the certificate is signed by the $\mathrm{CG}(\operatorname{Sig}(C G))$.

Observe that $\operatorname{Cert}(f)$ defined above is a certificate of authenticity for $f$ : it walks with the file, it is signed by a trusted authority, and it contains an identifier $i d_{f}$ that can be used for verifications (e.g., against fake file phenomenon) and integrity checks.

Even before that $L S$ expires, the owner can update the resource and ask for another certificate. For example, she may want to publish a re-edited version of a previously released song, or adding/removing some delegate(s) from the $D L$ list. Of course, eldest copies of the file can still circulate until the $L S$ expires; in fact, it is preferable for an owner to set moderately short life spans (e.g., one month), and regularly update the certificate, even if no declared field changed. However, the update request can have the same message format of the certification one: if the $\mathrm{CG}$ observes that a file with $i d_{f}$ have been previously deposited, then it checks if the copyrighted owner is the same. In such a case, it accepts the requests and issues a new certificate.

\section{The Cost of an Object}

The main idea of FairPeers is that when a peer downloads an object, then he should pay both the owner and the provider. We have three scenarios: (1) the provider is the owner (or a delegate), (2) there is only one provider, (3) there are multiple providers, i.e., multi-source download. Before showing the protocol behavior in all of these cases, we look at the $\mathrm{CG}$ and the copyright certificate in detail. 
The costs for distribution and for authoring a given object are substantially different. This is not the place for making a cost estimation, because it depends on several criteria, most of them based on the market trends.

Undoubtedly, the distribution cost can be quantitatively defined in a more precise way, if we accept that it is strongly related to his byte length: the more the file is big, the more bandwidth is consumed and storage is used. Anyway, we can assume that each file $f$ has two costs: an authoring cost $C_{a}(f)$ and a distribution cost $C_{d}(f)$. Hence, the total cost of fis $C(f)=C_{a}(f)+C_{d}(f)$, which is exactly the price that the user should pay for gaining it. For example, a mp3 song $s$ can have a cost of $1 \$$, where $C_{a}(s)=0.99 \$$ and $C_{d}(s)=0.01 \$$; a divx movie $m$, can costs $6 \$$, where $C_{a}(m)=5.50 \$$ and $C_{d}(s)=0.50 \$$. Anyway, in the rest of the paper we will assume that $C_{a}(f)$ and $C_{d}(f)$ are always deducible from $\operatorname{Cert}(f)$ that contains also the value $v$ imposed by the owner of $f$.

In the remaining of the paragraph, we describe the behavior of peers joining the network, without any assumption about the payment module that can be adopted. However, it should be observed that even if some authoring cost can be discharged by means of some macro-payment system (e.g., PayPal), the most part of the payments are due to low value objects (e.g., documents, mp3 songs) and distribution costs. As a consequence, these costs must be managed by efficient and scalable micro-payment schemes (e.g., PPay, PeerMint), that have to stress the central entities, like the bank and the brokers, as little as possible. In the following, we will describe each economic transactions with sentences like " $p e e r X$ credits peer $Y$ for value $c$ ', without specifying how the payment will be performed. For the sake of simplicity, we can think that such an operation takes place by transferring an electronic coin with value $c$ from $X$ to $Y$, or more precisely, using a call to pay $\left(i d_{x}\right.$, $\left.i d_{y}, \mathrm{c}\right)$, i.e., the payment primitive described in section service model.

\section{Purchasing an Object from The Owner}

This is the simplest case and likely the most uncommon. Let $A$ be the owner of file $f$ (or also a delegate for $f$ ), and $X$ the peer wishing to purchase $f$. We assume that peers communicate using authenticated channels, e.g., during session's handshake, peers exchange each other their public key certificates.

(a) $X \rightarrow A:\left\{\right.$ "I want id ${ }_{f}$ ' $\} \operatorname{Sig}(\mathrm{X})$;

(b) $A \rightarrow X:\{\operatorname{Cert}(f)\} \operatorname{Sig}(\mathrm{A})$;

(c) $X \rightarrow A$ : $\left\{\right.$ credits $A$ for $\left.C_{a}(f)\right\} \operatorname{Sig}(\mathrm{X})$;

(d) $A \rightarrow X:\{$ License $(f, X)\} \operatorname{Sig}(\mathrm{A})$;

(e) $X \rightarrow A$ : $\left\{\right.$ credits $A$ for $\left.C_{d}(f)\right\} \operatorname{Sig}(\mathrm{X})$;

(f) $A \rightarrow X: f$.

The session is started by $X$, that contacts $A$ likely after a search for $f$. $X$ sends to $A$ a (signed under his own private key) request for object $f$ (step (a)). This message is signed because in case of a dispute, $A$ needs to prove that $X$ wanted to buy the file identified by $i d_{f}$. A replies back with the $f$ 's copyright certificate (step (b)). Reminding that the copyright certificate is an unforgeable message signed by the grantor, $A$ is proving to $X$ that she is the owner of $f$, and she is accepting to upload 
the file. Moreover, $X$ is able to determine the costs $C_{a}(f)$ and $C_{d}(f)$. If she does not accept these prices, she can break here the connection: nothing has been credited, and the file has not been sent. First of all, in step (c), $X$ covers the ownership cost (e.g., in our prototype, $X$ assigns a PPay coin to $A$ with value $C_{a}(f)$ ) and waits for the distribution license (step $(\mathrm{d})$ ). The distribution license has the following format:

$$
\operatorname{License}(f, X)=\left\{i d_{A}, i d_{X}, \operatorname{Cert}(f)\right\} \operatorname{Sig}(A)
$$

The utility of the distribution license is two-fold: (1) it works like an acknowledgment receipt, and (2) from now on, $X$ can show the license in order to prove that he has been correctly authorized for sharing the file. An important side effect of using licenses instead of DRM systems is that, even if the user loses the file, she can continue to prove that she is still a valid provider, and she can request a new copy paying only the distribution cost. Moreover, $X$ will continue to access to the content without expiration, as it is used in many novel DRMs. Finally, $X$ can pay for the distribution (step (e)), and download the file from $A$ (step (f)). Of course, the protocol can be interrupted intentionally or not before its completion. We will shortly analyze security drawbacks at the end of this section.

\section{Purchasing an Object from a Provider}

After buying $f$, a peer is allowed to further upload it to other peers: now it is a provider for $f$. The file management module will announce the system that the searched object is shared by on-line providers and delegates (see section peer's architecture and implementation). Let $X$ be a peer looking for object $f, Y$ the only peer that replies to the search query, and $A$ the owner of $f$ (or a delegate that is online at that moment). The protocol changes a little bit, because $Y$ can provide distribution, but he cannot gain any ownership credits.

(a) $X \rightarrow Y:\left\{\right.$ "I want id ${ }_{f}$ ' $\} \operatorname{Sig}(\mathrm{X})$;

(b) $Y \rightarrow X:\{$ License $(f, Y)\} \operatorname{Sig}(Y)$;

(c) $X \rightarrow A$ : $\left\{\right.$ credits $A$ for $\left.C_{a}(f)\right\} \operatorname{Sig}(\mathrm{X})$;

(d) $A \rightarrow X, Y:\{$ License $(f, X)\} \operatorname{Sig}(\mathrm{A})$;

(e) $X \rightarrow \mathrm{Y}:\left\{\right.$ credits $\mathrm{Y}$ for $\left.C_{d}(f)\right\} \operatorname{Sig}(\mathrm{X})$;

(f) $Y \rightarrow X: f$.

Message (a) remains unchanged. In message (b) $Y$ sends the distribution license to $X$, that includes the copyright certificate. Message License $(f, Y)$ proves that $Y$ was allowed by the owner to sell the object. However, now $X$ knows who is the owner (and who are the delegates), and that $Y$ is an "official" provider. At this step, $X$ should check if the owner is on-line or if it is ready to receive a payment (e.g., by way of a ping message). If the owner is not available, a delegate will be contacted. Note that, if it is impossible to contact the owner or the delegate, the copyright grantor can accept payments and produce the license. If the CG is a central server, it can debit the owner for this outsourcing service.

Let $A$ be the owner or the given delegate that will cash the ownership payment in step (c). Then, $A$ will produce a new license for $X$. Notice that step (d) is the first real 
difference w.r.t. the simple case: the owner will send a copy of the new license to the provider $Y$ for synchronization purposes; after receiving License(f,X), $Y$ is authorized to upload the file, and $X$ is advised that $Y$ gained the license. Steps (e) and (f) remain the same.

\section{Purchasing an Object from Many Providers}

This is the most common scenario: when an object is popular, many peers want to download it, and many providers are available. The protocol seen in the previous paragraph can be generalized in a quite straightforward way: distribution cost $C_{d}(f)$ is divided for $m$, where $m$ is the number of segments of $f$ (e.g., a file of $1 \mathrm{MB}$ can be divided in 10 segments of $102,4 \mathrm{~KB}$ ). Given a receiver $X$, each provider $Y_{i}$ is credited for a value equal to $m_{i} \frac{C_{d}(f)}{m}$, where $m_{i}$ is the number of segments that $Y_{i}$ served to $X$. Unfortunately, this solution has many drawbacks. First of all, scalability is an issue: in fact, each download will involve many participants, and the number of overall economic transactions would grow of some order of magnitude. If the payment module uses some central authorities (e.g., the broker in PPay), the efficiency of the entire system would decrease. Moreover, the more micro-payment tokens or messages with legal value flow in the system, the more the number of frauds (e.g., double spending) grows. This is acceptable if the overall number of transactions grows with the risks, but in this scenario a receiver should perform many crediting operations for only one download. The last, but not the least, problem is that each transaction is valued in the order of fractions of one cent: does it make sense to deal with a complex system that manage pico-payments?

We decided to introduce in FairPeers an efficient lottery policy: instead of paying a fraction of $C_{d}(f)$ to all the providers, only a winner will gain the entire revenue for the distribution. The issuer of the lottery (i.e., the receiver) will always pay the distribution cost. The risk for the peers is that too few of them will turn out to be winners. As discussed by Rivest in [45], the law of large numbers gives comfort: after many lotteries, the expected value of the payment is approximately correct, with a low variance. For example, lets consider a peer that shares a very popular resource, with a distribution cost of 1 cent. In a given slot of time, he uploads one of the parts of the given object for 1000 times. If we have an average of 10 participants to the multi-download sessions, he should collect at each upload the amount of 0.1 cent. Hence, at the end of the observed period he has gained $0.1 \cdot 1000$ cents $=$ $1 \$$. Lets see the lottery case: he has a probability 0.1 to win 1 cent at each session. After 1000 transactions, the expected value is exactly $1 \$$.

Let $\bar{Y}=\left\{Y_{1}, \ldots, Y_{n}\right\}$ be the set of $n$ providers of $f$, and $X$ be the receiver. For the sake of simplicity, let us assume that each provider $Y_{i}$ provides only one segment $f_{i}$. The first steps ((a)-(d)) do not change, except for the set $\bar{Y}$ that substitutes $Y$. Steps from (e) are as follows:

(e) $X \rightarrow \bar{Y}:\{\bar{Y}$, “winner indicator', $\} \operatorname{Sig}(\mathrm{X})$;

(f) $\forall \mathrm{Y}_{i} \in \bar{Y}, Y_{i} \rightarrow X: f_{i}$;

(g) $X \rightarrow \bar{Y}:\left\{\right.$ " the winner is $Y_{w}$ "' $\} \operatorname{Sig}(\mathrm{X})$;

(h) $X \rightarrow Y_{w}:\left\{\right.$ credits $Y_{w}$ for $\left.C_{d}(f)\right\} \operatorname{Sig}(\mathrm{X})$. 
Before running step (e), $X$ knows exactly the members of set $Y$, and their identifiers $i d_{1}, \ldots, i d_{n}$. She can pick one of these identifiers $\left(i d_{w}\right)$ at random, and proclaim $Y_{w}$ as the winner. $X$ does not communicate the identity of the winner before receiving the $n$ segments; instead, she issues a winner indicator, that can be used by the providers to verify who is the winner. The winner indicator $h^{\prime}$ is the result of $h\left(i d_{w} \oplus \mathrm{s}\right)$, where $s$ is a random salt generated by $X$, and $h$ is a collision resistant hash function (e.g., SHA-1). When provider $Y_{i}$ receives the winner indicator, he starts uploading the agreed segment $f_{i}$. Step $(\mathrm{g})$ occurs when all the segments have been correctly uploaded: $X$ broadcasts the value of $s$, allowing every provider $Y_{i}$ to determine if they are the winner or not (i.e., if $h^{\prime}=h\left(i d_{i} \oplus \mathrm{s}\right)$, then $\left.i d_{i}=i d_{w}\right)$. Only the winner is credited for $C_{d}(f)$ in step (h). Note that $X$ broadcasts all the participants' identifiers to the providers in step (e). This is due to allow everybody to further check if there is at least a winner of the lottery.

\section{Security Analysis and Reputation Management}

In this domain we can potentially have different peers that behave maliciously independently or in accordance. Some remarks and discussions are in order.

\section{Trivial Cheating}

This group of frauds assumes that a given instance of the protocol is not interrupted during its execution. Peer $A$ may try to cheat by asserting he is the owner (or a delegate) of a content he did not create (or he has not been delegated to represent). In this case, note that, in order for $A$ to be successful, he should have been able to forge a valid copyright certificate (i.e. a signature) with respect to the CG's secret key. This is clearly infeasible if one assumes that the underlying signature scheme is existentially unforgeable against adaptive chosen message attacks (see [47] for details).

Another way for $A$ and/or $Y$ to cheat a receiver, is claiming they never interacted with $X$ (and thus that $X$ holds the content illegally). But they cannot claim they did not interact with customer $X$, because they actually sent a signed message in response to $X$ 's request for $f$.

\section{Protocol Aborting}

A trivial observation is that a malicious customer $X$ cannot refuse to pay the owner or the provider for a content she received simply because the owner sends the license only after he has been correctly credited (as well as the provider that sends the file after receiving the payment). Similarly $X$ cannot pretend she did not want to buy $f$ (see discussion on step (a) above). An obvious way on which an owner or a delegate may try to cheat is by aborting the protocol upon being credited for $C_{a}(f)$ (between steps (c) and (d)). Consider that we request to the underlying payment layer that $\mathrm{X}$ can always prove that she actually paid the requested object. $\mathrm{X}$ can present the payment's receipt (or the token he forged) to a trusted third party 
attention (e.g., the Broker in PPay, or another honest peer in the system, see discussion in paragraph "Who to blame in case of fraud?"). A can repair to the actions against him sending the license or even the file (again), without any further additional costs. Observe that both $X$ and $A$ get no benefit in cheating each other: if $A$ says that she did not receive the payment, she will receive back the same payment (e.g., the assigned coin) already forged for her. However, she will be able to spend this amount of currency only once (this is equivalent to double spending, which is a traceable - and punishable!-activity in the underlying payment scheme). On the other hand, if $X$ received the object (i.e., the license) and claims that $A$ cheated (or that the connection went down during download), he will receive just a copy of License $(f, X)$. Same discussion applies when the provider (or the owner) aborts the protocol described in section purchasing an object from a provider (or purchasing an object from the owner) upon being credited for $C_{d}(f)$ (between steps (e) and (f)).

The lottery scenario (section purchasing an object from many providers) is a little bit more complicated: the receiver credits the winning provider after receiving the file. Assuming at the moment no collusions between peers, we can consider two different cases: (1) one (or more than one) provider(s) breaks uploading the file at step (f), and (2) the customer aborts the protocol after step (f). The first case can be repaired as follows: the leaving player $Y_{l}$ is left out by the lottery, and another peer will provide file segment $f_{l}$. Maybe there are many ways for implementing this. Our solutions is to release another ticket, and start again from step (e), i.e., a new pair $\left\langle i d_{w^{\prime}}, s^{\prime}\right.$ is created, and message $\left[h\left(i d_{w} \oplus s\right), h\left(i d_{w \prime} \oplus \mathrm{s}^{\prime}\right)\right]$ is broadcasted to "surviving' $Y_{i}$. Of course, there is no need to delete correctly delivered file's segments. ${ }^{1}$ When the winner is proclaimed, the new salt can be sent together to the rejected winner indicator(s), for a final verification, i.e., message in step (g) becomes $\left[h\left(i d_{w} \oplus s\right), \mathrm{s}^{\prime}\right]$. All the peers will store these messages for off-line verifications for a predefined period of time (e.g., a week).

In the second case, $X$ stops the protocol after downloading. If she breaks the connection after communicating the winner $Y_{w}$, then it trivially follows like in the previous case: $Y_{w}$ has the proof that $X$ must credit him. Otherwise, if $X$ breaks exactly between steps (f) and (g), then the problem is more subtle. In fact, we have to distinguish two more cases. When the winning salt $s$ reaches only a subset of $\bar{Y}$, a peer, after a given timeout, can request message (g) signed by $X$ to random selected group of other providers (this is why $X$ at step (e) must include $\bar{Y}$ identifiers). Conversely, when $X$ does not send $s$ to anyone, the following strategy can be adopted: a life span is assigned to each lottery, and when the time is over, and no provider has obtained a response (they keep informed each other after short-period timeouts), then the lottery is marked "not valid". Hence, each $Y_{i}$ can take this anomalous behavior to a third party attention. Finally, in both cases, replay attacks (e.g., a rival provider can send the outcome of another lottery) can be avoided by including in each exchanged messages a time stamp or a session identifier assigned to each different lottery.

\footnotetext{
1 Note that this approach can be iterated and also be used when a new provider wants to join the lottery (and delivering his own file's segment).
} 


\section{Collusions}

There are different kinds of possible collusions. First of all, receivers and providers can try to behave accordingly in order to cheat the owner: in fact, $Y$ can send to $X$ the file without contacting owner $A$, and after having negotiated another price. But, in this case, $X$ owns an object licensed for $Y$. As a consequence, $X$ cannot prove to a third party that he is legally holding the given object. ${ }^{2}$ Of course, consider that such a protocol does not cope with dishonest peers that cooperate in order to cheat owners, outside the discussed scheme (for example $Y$ could burn an audio CD with all the songs performed by $A$ and sell it to $X$ at a lower price when they meet at the pub). This is a DRM problem and is out of the scope of this proposal, even if we think that an incentive scheme is much more promising that a punitive policy. In general, FairPeers is very protective w.r.t. customers: it is very difficult to cheat a receiver, because they can immediately react without paying. Moreover, as we discussed, providers can always prove if a customer cheated in the first two versions of the protocol. But during a lottery, a provider can be in troubles with a collusion between the receiver and other providers. In fact, they can negotiate a fake winner in advance, and use the collaboration of another honest provider for gaining missing file's segments for free. From the point of view of the honest provider, this is not a real fraud: it is like losing to a lottery. But this selfish strategy can be discouraged if all the peers maintain a list of won/lost lotteries, with customers' identifiers: they can give low priority to receivers that managed not profiting multi downloads sessions.

\section{Sybil Attack in the Lottery Scheme}

A peer performs a Sybil attack [48] when he appears in the system under different identities. In the lottery scheme this can be a problem if a customer $X$ downloads a file's segment from one honest provider, and from $n-1$ other fake peers, that are different aliases of $X$ himself. If this cheat is repeated other $n-1$ times, with different parts of the desired file, $X$ can obtain the resource without paying distribution costs.

This can be considered as a sophisticated kind of collusion, but in this case deceived peer $Y_{i}$ has a chance to detect the fraud; in fact, he can send a challenge to another (randomly chosen) $Y_{j}$ in $\bar{Y}$, which should be responsible of serving part $f_{j}$ to $X$. $Y_{i}$ can send a random bit sequence $r$ to $Y_{j}$, asking for an hash of $\left(f_{j} \oplus r\right)$. If $Y_{j}$ has that part, then he can reply with the correct value. $Y_{i}$ can easily verify because he has the whole file. Otherwise, the challenge fails, and $Y_{i}$ leaves the lottery. Observe that, in any case, this strategy is interesting in a competitive market.

\section{Who to Blame in Case of Fraud?}

We have seen that frauds are possible, but cheated peers can prove when they take place. When a peer can bring another peer's misbehaving activity to a trusted third

\footnotetext{
${ }^{2}$ In a practical implementation of such a proposal, periodic and random tests should be performed over objects owned by the peer, in order to check against their license validity.
} 
party attention, we have to define who plays this role. When a central entity exists (e.g., the broker in PPay), the answer appears to be clear. However, if a fraud occurs during a macro-payment transaction, the cost of denouncing the event is justified. Conversely, when the cheat consists of few cents (or even less), contacting a trusted third party appears to be an inefficient and pointless solution. Our opinion is that a reputation (centralized or decentralized) mechanism should be used instead when a micro-payment fraud has been detected. Moreover, the provability of frauds is very useful in a reputation system: when a peer wants to trade with another peer, he may ask for the credibility degree the latter earned. In general when a (negative) feedback is given, there is no chance of asking for a reliable reason (e.g., feedback rating in eBay). In FairPeers, a peer can ask the prove of a given denounced fraud (by means of a bad assigned reputation rate) to the cheated user. Then, he can decide to go on (at his own risk, in the case the negative feedback has a real foundation) with the given trading. Moreover, the peer accused to have provoked the fraud can repair: in this case the negative feedback can be recused by the issuer. Finally, consider that when a peer received an unjustified negative feedback (i.e., a false alarm), then he can always to counter proof his innocence.

Of course, proofs older than a given period (e.g., one week or one month) can be deleted. However, it is easily predictable that a reputation system, enforced by provability of negative feedbacks, would greatly reduce illicit behaviors and false alarms in a self-organizing and efficient way.

\section{Simulations}

In this section, we present experimental results obtained from a simulation of a market-oriented scenario in which agents trade items each other mimicking the real world. The experiments are devoted to support the motivations behind the adoption of the FairPeers proposal and, with reference to the framework described in section service model, they only take into account the components involved at the Market Level. In relation to the prototype architecture described in section peer's architecture and implementation, some efficiency considerations about the Pastry protocol adopted (Overlay Level) can be found in [37]. Moreover, a scalability evaluation of the PPay accounting scheme in a FairPeers-enabled market (Accounting Level) has been deeply discussed in [43]. However, in the following, we focus on the fairness and the incentive-compatible nature of the proposal.

To model the marketplace, we imagine a scenario in which a set of $m$ distinct objects, $f_{1}, f_{2}, \ldots, f_{m}$ are shared by a population of $n$ agents $a_{1}, a_{2}, \ldots, a_{n}$ behaving as providers, or receivers, or both. The popularity of the objects in the system follows a Zipf's law as observed in [7, 49]. We suppose that every object is composed by $B$ different file blocks with $B$ uniformly distributed in the interval $\left[B_{M I N}, B_{M A X}\right]$. If an agent holds an object $f_{i}$, we assume that it owns all the other file blocks. Accordingly, the model allows the standard multi-source download scheme implemented in modern file-sharing applications. At each step, an agent can simultaneously manage the upload of up to $Z$ file blocks by way of a finite upload queue of $Z$ slots. 
During a simulation run, a generic agent $a_{i}$ receives a file according to a download ratio $\delta \in[0,1]$. On the other side, the contacted agent decides whether to provide or not the requested content according to a upload ratio $\mu \in[0,1]$. We define different levels for $\delta$ and $\mu$ and the combination of such values defines the strategy that an agent pursues. In particular, we identify four types of provider's strategies in relation to the degree of contribution:

- Egoistic: an agent that performs only downloads without sharing his resources $(\mu=0)$. It mimics the free-rider behavior described in section background and related work.

- Altruistic: an agent that, if a slot is available, always provides the requested block $(\mu=1)$.

- Moderate: it follows a balanced strategy in which it acts half as egoistic and half as altruistic $(\mu=0.5)$.

- Greedy: it contributes in a small fraction of upload requests $(\mu=0.25)$.

After these premises, we define the download ratio $\Delta=\frac{\text { down }}{\text { down+fail }}$, where down is the number of successful download and fail is the number of requests that have been rejected due to a deliberate agent's choice (low value of $\mu$ ) or an unsustainable incoming traffic (slots queue full). In the first part of this analysis, we wanted to graphically show the utility function $\Delta$ in several scenarios characterized by an increasing level of contribution, i.e. a growing fraction of altruistic agents, with respect to the dimension of the upload queue. As expected, Fig. 2 draws two major effects. On the one hand, the download ratio $\Delta$ is related to the agent's contribution in terms of the number of slots it makes available (each curve exhibits a rising trend). On the other hand, the balance between altruistic and egoistic behaviors heavily affects the efficiency of the market: the success of a download depends on the agent's voluntary choice to share its own resources. It is worth noting that we run several simulations with an increasing number of agents and we collected the same results (in Fig. $2 n$ is equal to 5000), indicating that the previous observations are valid independently to the agents' population size.

Fig. 2 Download ratio $\Delta$ in different scenarios characterized by an increasing degree of contribution ( $e$ and $a$ represent respectively the fraction of egoistic and altruistic agents)

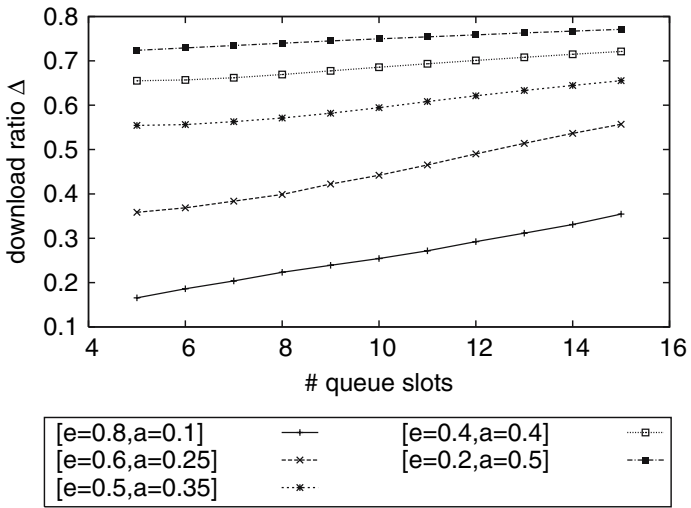


Fig. 3 Average gain in a standard or in a FairPeers-enabled market due to the distribution of content

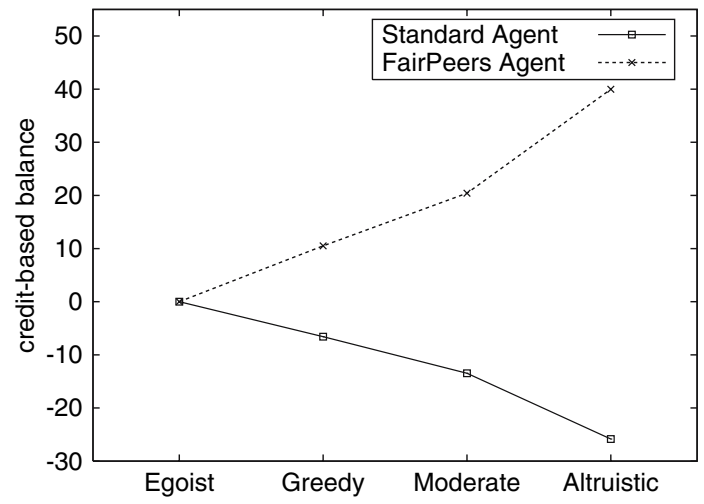

The next part of the analysis is intended to study the relation between costs and rewards in two different scenarios: (1) a marketplace in which the agents trade objects following the standard file-sharing mechanisms and (2) a marketplace in which agents follow the FairPeers protocol. In the first case, a provider is charged by a cost $C_{\text {upload }}$ for each file blocks uploaded due to the amount of resource provided, e.g. bandwidth, CPU cycle, disk space. In the latter case, together with the upload cost, each download corresponds to a twofold monetary transfer: $C_{a}$ is paid to the item's owner and $C_{d}$ to the lottery's winner. Figure 3 shows the average gain or loss with respect to the different provider's strategies. In the standard scenario, the freerider phenomenon (see section background and related work) observed in several previous studies [50, 7] is evident: the revenue of a generic agent decreases according to the growth of the altruism degree. In other words, a rational agent is pushed toward an egoistic conduct which minimizes the costs.

In a FairPeers-enabled market the situation is specular: since the distribution of content is rewarded, the altruistic agents obtain the greatest gain. The consequences are multifarious: firstly, FairPeers represents an incentive-compatible way to stimulate cooperation among peers. In fact, an altruistic agent obtains the best payoff. Moreover, the proposal is not only a technical mechanism to avoid freeriding, but it proposes a business model in which agents are able to gain a revenue in relation to their level of contribution. At last, the fairness is guaranteed because both the owners of the content as well as the providers are refunded. A relevant difference between FairPeers and a standard centralized music store, e.g. iTunes, is essentially the ability for the peers to legally redistribute a content previously bought without any form of DRM systems and, above all, the consideration that the distribution process is economically profitable for participants.

\section{Peer's Architecture and Implementation}

A prototype environment of the FairPeers' marketplace has been implemented in Java for testing and emulation purposes. The architecture at the peer's side can be divided in the following major components (Fig. 4): File Management, Payment 




Fig. 4 General architecture of FairPeers prototype

Management and Reputation Engine that is transversal to both the previous ones. We give a brief "nuts and bolts view" of each of these functional modules.

File Management

File Management implements two basic features: (1) Certification and Publishing, and (2) Content Management.

\section{Certification and Publishing}

When an owner creates an inedit content, he needs to register it at the Copyright Grantor in order to generate the certificate that binds the identity of the owner with the related content. The module Publishing in the scheme is intended to achieve this goal. It is important to underline that the actual implementation uses a central server to manage the copyright certificate generation and storage. Of course, storaging could be implemented in a distributed manner without affecting the overall architecture. Furthermore, this module attends to the validity checks of provider's or owner's licenses, along with the storage and management of copyright-related information. It uses directly the underlying Internet to communicate exploiting the Authentication module in order to deal with cryptographic operations. 


\section{Content Management}

It provides the high-level functionalities present in a standard file sharing system, i.e., insertion, discovery and download of resources. In order to implement a file sharing layer, the prototype makes use of a customized version of the distributed storage service PAST [51], called in the figure PAST ${ }^{*}$, that is build on top of Pastry [37].

The choice of Pastry as overlay network is due to its main features: a scalable routing mechanism, a caching and replication schemes that ensure an adequate level of reliability, along with the availability of an open source implementation, i.e. the FreePastry API [52], that provides basic authentication and security functionalities. Notwithstanding, any other structured overlay network could be successfully applied, e.g., Chord [38] or Kademlia [39]. Furthermore, the adoption of an unstructured approach, like Gnutella [36] or KaZaA [22], could depict an alternative solution characterized with a smaller degree of scalability.

Starting from the consideration that a distributed storage service tries to fulfill a set of requirements slightly different from a file-sharing application, we have customized the PAST layer instead of implementing from scratch a novel storage module. A main characteristic of this module is the concept of reference. The PAST storage layer does not bind identifiers directly to a resource, but with a reference to a resource. In the prototype a reference contains all the information needful to locate the entities involved in a download: the set of sources, the owner (along with the delegates if they are specified), and other additional metadata. In other words, the module PAST ${ }^{*}$ does not store files but references to files, according to a mechanism called indirect storage. This layer is able to provide a set of references matching the searching criteria, whereas the download process is managed by means of direct connections through the underlying network communication layer. In the rest of the section, we will sketch the key concept regarding the insertion, the search and the download of files.

\section{Insertion Mechanism}

Whenever a user shares a file, a insert function is invoked. Firstly, the power set of the words that compose that file name is calculated, discarding the special characters like punctuation marks. Then, for each subset, a string, with the words in alphabetical order separated by a blank, is generated. The hash of these formatted strings is used as identifier in an insertion message submitted to the PAST ${ }^{*}$ layer. For example, given the file "Beatles-Yesterday.mp3"' we compute the corresponding power set composed by the formatted strings \{Beatles\}, \{Yesterday\} and $\{$ Beatles, Yesterday\}. At last, the hash of each string is used as identifier and inserted in the storage with the related reference to the resource. A similar approach is implemented in eMule [16], that is based on Kademlia [39].

\section{Searching Mechanism}

In a file sharing system, a user specifies a set of keywords that describes the resource he is looking for, and he receives the references to files matching the search criteria 
along with the addresses of nodes that store them. The prototype allows a user to submit a query string, that is managed through the same algorithm used in the insertion protocol, in order to guarantee convergence between insertion and searching.

\section{Download Mechanism}

From a technical point of view, the download process is implemented by means of a direct file transfer between the sources and the querying peer, and TCP sessions are established off the overlay network. In fact, the distributed storage service manages pointer to resources, but resources are maintained to the owner's or provider's side. Download is implemented with interactions with the payment management module, because of the FairPeers' definition (Section 4). In Fig. 4, there is a tight relation between the accounting functionalities and the file sharing module.

\section{Payment Management}

The goal of the Payment Management is to provide a secure and efficient payment and accounting mechanisms able to manage the economic transactions between peers.

The FairPeers prototype provides a Java implementation of PPay [53]. Basically, the PPay protocol is based on the concept of floating and self-managed currency. The coins can float from one peer to another, and the owner of a given coin manages the currency itself, except when it is created or cashed. As other micropayments systems, also in PPay some coin fraud is possible, but it is unprofitable. Frauds are detectable by means of the involvement of a trusted third party, the Broker. It directs, in a centralized manner, the printing and the cashing of coins. Moreover, it stores the account balance of users and detects the forgery and double spending frauds. It is clear that the broker reduces the overall scalability of the payment component because it could be a single-point-failure in the presence of an high volume of peers and transactions, in spite of an increased efficiency and security level. An analysis of the configuration parameters that guarantee an efficient implementation of PPay protocol is presented in [43]. In addition, an original use of aggregate signatures is described in [54], that allows the reduction of floating coins verification costs.

In any cases, since FairPeers is independent from the underlying payment substrate, we could adopt other solutions without affecting the system's properties. Several fully decentralized approaches can be found in $[44,55,56]$.

\section{Reputation Engine}

A leading role in the FairPeers architecture is played by the Reputation Engine: as described in section background and related work a reputation scheme is an effective way to counter the misbehaving of peers. The Fig. 4 shows that this module is strongly related to the other components of the prototype providing a reputation mechanism at different levels: 
- Overlay level: A peer may not forward the searching requests or he can maliciously not provide the content (i.e., the pointer to a resource) that he is responsible for. The phenomenon becomes worse in a competitive environment like a market place where peers trade services each others: a peer acting in its own best interest will not forward queries to potential competitors [57].

- Accounting level: Forgery or double spending frauds are possible in PPay, even if third parties are able to trace back them.

- Market level: A user can break maliciously the FairPeers protocol in any step, for example, after the upload of the desired content and before the payment is performed. Even if this actions are provable, we need to take countermeasures against this malicious behaviors (see discussion in section security analysis and reputation management).

The present prototype uses a unique trusted third party, i.e., the Broker, for solving disputes. The introduction of a reputation module is in our plans. This engine should be able to push the user toward a correct behavior without implementing rigid tracing and punishment policies. As underlined in section security analysis and reputation management, at the market level, the effectiveness of the reputation module is enforced by the provability of negative feedbacks. Other simple reputation schemes have successfully been used at the accounting and overlay levels [58]. Of course, a final reason for implementing a reputation module is that scalability is strongly improved, because of the viral dissemination of feedbacks, and the reduction of stressing load at the broker side. It is important noting that the architecture of the reputation module is not strictly part of the FairPeers core proposal. Thereafter, its implementation can take inspiration from one of the approaches present in the reputation-related literature (see [59-61] for a survey).

\section{Lessons Learned}

The experience learned through the implementation of the FairPeers prototype lead us to delineate some useful considerations and suggestions in order to build real Peer-to-Peer market places. Firstly, it is worth noting that, actually, the Peer-to-Peer paradigm and the tools available are pretty mature to allow a modular and complete engineering of all the different components of a market application. However, a lot of work has to be put in place in the future. For instance, the current FreePastry and PAST APIs need a personalization (as we made with PAST ${ }^{*}$ ) in order to build a reliable and efficient content distribution platform. Moreover, the security primitives provided by these libraries lack to a strong level of authentication and access control functionalities. Future distributions of such APIs must cover these functions and security management primitives as "built-in".

\section{Conclusions and Future Works}

We have presented FairPeers, that according to the service model presented in section service model, is the only $\mathrm{P} 2 \mathrm{P}$ market framework that guarantees both 
fairness and copyright management. Moreover, a peculiar multi-source distribution mechanism is based on a lottery scheme, that maintains fairness without invalidating typical P2P efficiency.

A prototype architecture and implementation is based on PPay, a micro-payment system that has been proved efficient, secure and reliable. Such an environment can be used for testing different economic (malicious and legal) strategies that peers should follow in order to increase their profit.

Future works include the integration of a reputation module that will also increase scalability (i.e., the central broker's assistance will not be requested at each dispute). Moreover, FairPeers can be used for implementing time sensitive applications like streaming, because application level multi-cast trees can largely benefit of the multi-source distribution scheme described above.

Acknowledgements This work has been partially supported by the Italian Ministry for University and Research (MIUR), within the framework of the "PROFILES', project (PRIN). The authors would like to thank the anonymous referees for their insightful comments about how to improve this work. Furthermore, we would like to thank Burkhard Stiller and David Hausheer for the profitable discussions we had at early stages of our studies.

\section{References}

1. Steinmetz, R., Wehrle K. (eds.): Peer-to-Peer Systems and Applications. LNCS 3485. Springer (2005)

2. Chuang, J., Feldman, M., Lai, K., Stoica, I.: Quantifying disincentives in peer-to-peer networks. In: First Workshop on Economics of Peer-to-Peer Systems, UC Berkeley, Berkeley, California, USA (2003)

3. Habib, A., Chuang, J.: Incentive mechanism for peer-to-peer media streaming, June 2004

4. Adar, E., Huberman, B.: Free riding on gnutella. Technical report, Xerox PARC (2000)

5. Jobs, S.: Thoughts on music. http://www.apple.com/hotnews/thoughtsonmusic/, Febraury 2007

6. Ruffo, G., Schifanella, R., Ghiringhello, E.: A decentralized recommendation system based on selforganizing partnerships. In: IFIP-Networking, vol. 3976 of Lecture Notes in Computer Science, pp. 618-629. Springer (2006)

7. Saroiu, S., Gummadi, K.P., Gribble, S.D.: A measurement study of peer-to-peer file sharing systems. In: Multimedia Computing and Networking (MMCN), January 2002

8. Hughes, D., Coulson, G., Walkerdine, J.: Free riding on gnutella revisited: The bell tolls? IEEE Distributed Systems Online 6(6), 1 (2005)

9. Shneidman, J., Parkes, D.C.: Using redundancy to improve robustness of distributed mechanism implementations. In: EC '03: Proceedings of the 4th ACM conference on Electronic commerce, pp. 276-277. ACM Press, New York, NY, USA (2003)

10. Camerer, C.: Behavioral game theory: Experiments in strategic interaction. Princeton University Press, March 2003

11. James Andreoni.: Giving with impure altruism: Applications to charity and ricardian equivalence. J. Polit. Econ. 97(6), 1447-1458 (1989)

12. Andreoni, J.: Impure altruism and donations to public goods: A theory of warm-glow giving? Econ. J. 100(401), 464-477 (1990)

13. Feldman, M., Papadimitriou, C., Chuang, J., Stoica, I.: Free-riding and whitewashing in peer-to-peer systems. In: PINS '04: Proceedings of the ACM SIGCOMM workshop on Practice and theory of incentives in networked systems, pp. 228-236. ACM Press, New York, NY, USA (2004)

14. Bittorrent project. http://www.bittorrent.com

15. Cohen, B.: Incentives to build robustness in BitTorrent. In: 1st Workshop on Economics of Peer-toPeer Systems, June 2003

16. Kulbak, Y., Bickson, D.: The emule protocol specification (2005) 
17. Shirky, C.: The case against micropayments. OpenP2P O'Reilly, December 2000

18. Odlyzko, A.: The case against micropayments. In: 7th International Conference of Financial Cryptography, LNCS 2742. Springer (2003)

19. Fishburn, P.C., Odlyzko, A.M.: Competitive pricing of information goods: Subscription pricing versus pay-peruse. Econ. Theory 13(2), 447-470 (1999) Available at http://www.ideas.repec.org/a/ spr/joecth/v13y1999i2p447-470.html

20. Bakos, Y., Brynjolfsson, E.: Bundling information goods: Pricing, profits and efficiency. Working Paper Series 199, MIT Center for Coordination Science, January 1997. Available at http:// www.ideas.repec.org/p/wop/mitccs/199.html

21. Mojo Nation (currently named Mnet) project. Available at http://www.en.wikipedia.org/wiki/mnet

22. KaZaA. KaZaA Web Site. Available at http://www.kazaa.com

23. Osborne, M.J., Rubinstein, A.: A Course in Game Theory. The MIT Press, July 1994

24. Buragohain, C., Agrawal, D., Suri, S.: A game theoretic framework for incentives in p2p systems. In: P2P '03: Proceedings of the 3rd International Conference on Peer-to-Peer Computing, p. 48. IEEE Computer Society, Washington, DC, USA (2003)

25. Golle, P., Leyton-Brown, K., Mironov, I.: Incentives for sharing in peer-to-peer networks. In: EC '01: Proceedings of the 3rd ACM conference on Electronic Commerce, pp. 264-267. ACM Press, New York, NY, USA (2001)

26. Chuang, J., Feldman, M., Lai, K., Stoica, I.: Incentives for cooperation in peer-to-peer networks. In: First Workshop on Economics of Peer-to-Peer Systems, UC Berkeley, Berkeley, California, USA (2003)

27. Krishnan, R., Smith, M.D., Tang Z., Telang R.: The impact of free-riding on peer-to-peer networks. In: Proceedings of the 37th Annual Hawaii International Conference on System Sciences-Track 7. IEEE Computer Society, Washington, DC, USA (2004)

28. Blanc, A., Liu, Y., Vahdat, A.: Designing Incentives for Peer-to-Peer Routing. In: Second Workshop on the Economics of Peer-to-Peer Systems (P2PEcon), Harward University, Cambridge, MA, January 2004

29. Becker, J.U., Clement, M.: The Economic Rationale of Offering Media Files in Peer-to-Peer Networks. In: Proceedings of the 37th Annual Hawaii International Conference on System Sciences (HICSS'04) - Track 7, p. 70199.2, IEEE Computer Society, Washington, DC, USA (2004)

30. Antoniadis, P., Courcoubetis, C., Mason, R.: Comparing economic incentives in peer-to-peer networks. Comput. Networks 46(1), 133-146 (2004)

31. Feigenbaum, J., Shenker, S.: Distributed algorithmic mechanism design: Recent results and future directions. In: Proceedings of the 6th International Workshop on Discrete Algorithms and Methods for Mobile Computing and Communications. pp. 1-13. ACM Press, New York (2002)

32. Loginova, O., Wang, X.H., Lu, H.: Price peer-to-peer networks: A mechanism design approach. Working Papers 0608, Department of Economics, University of Missouri, July 2006. Available at http://www.ideas.repec.org/p/umc/wpaper/0608.html

33. Shneidman, J., Parkes, D.C.: Rationality and self-interest in peer to peer networks. In: Frans Kaashoek, M., Stoica, I. (eds.), IPTPS, vol. 2735 of Lecture Notes in Computer Science, pp. 139-148. Springer (2003)

34. Gerke, J., Hausheer, D., Mischke, J., Stiller, B.: An Architecture for a Service Oriented Peer-to-Peer System (SOPPS). Praxis der Informationsverarbeitung und Kommunikation (PIK) 26(2) (2003)

35. Hausheer, D.: PeerMart: Secure Decentralized Pricing and Accounting for Peer-to-Peer Systems. PhD thesis, Shaker Verlag, Aachen, Germany, ETH Zurich, TIK-Schriftenreihe No. 70, Diss. ETH Zurich No. 16200, March 2006

36. Gnutella Protocol Development Group. The Gnutella Protocol Specification 0.4/0.6. http://www.rfcgnutella.sourceforge.net

37. Rowstron, A., Pastry, P.D.: Scalable, distributed object location and routing for large-scale peer-topeer systems. In: IFIP/ACM International Conference on Distributed Systems Platforms (Middleware), pp 329-350. November (2001)

38. Stoica, I., Morris, R., Karger, D., Kaashoek, F., Balakrishnan, H.: Chord: A scalable Peer-to-Peer lookup service for internet applications. In: ACM SIGCOMM 2001. San Diego, CA, September (2001)

39. Maymounkov, P., Mazières, D.: Kademlia: A peer-to-peer information system based on the xor metric. In: IPTPS '01: Revised Papers from the First International Workshop on Peer-to-Peer Systems, pages 53-65. Springer-Verlag, London, UK (2002)

40. The Napster Web Site. http://www.napster.com 
41. Zhang, X., Liu, J., Li, B., Yum, T.-S.P.: Donet/coolstreaming: A data-driven overlay network for live media streaming. In: Proc. of IEEE INFOCOM'05. March 2005

42. Catalano, D., Ruffo, G.: A fair micro-payment scheme for profit sharing in a p2p network. In: Proc. of HOT-P2P 04. IEEE Press, October 2004

43. Ruffo, G., Schifanella, R.: Scalability evaluation of a peer-to-peer market place based on micro payments. In: Proc. of HOT-P2P 05. IEEE Press, July 2005

44. Hausheer, D., Stiller, B.: Peermint: Decentralized and secure accounting for Peer-to-Peer applications. In: IFIP Networking Conference, pp. 40-52. University of Waterloo, Waterloo, Ontario, Canada, May 2005

45. Rivest, R.L.: Electronic lottery tickets as micropayments. In: FC '97: Proceedings of the First International Conference on Financial Cryptography, pp 307-314, Springer-Verlag, London, UK (19970

46. Nikander, P., Karvonen, K.: Users and trust in cyberspace. In: Security Protocols Workshop, vol. 2133 of LNCS, pp 24-3. Springer (2000)

47. Micali, S., Goldwasser, S., Rivest, R.: A digital signature scheme secure against adaptive chosen message attacks. SIAM J. Comput. 17(2), 281-308 (1988)

48. Douceur, J.: The sybil attack. In: Proceedings of the IPTPS02 Workshop. March 2002

49. Iamnitchi, A., Ripeanu, M., Foster, I.: Small-world file-sharing communities. In: The 23rd Conference of the IEEE Communications Society (InfoCom 2004). Hong Kong (2004)

50. Golle, P., Leyton-Brown, K., Mironov, I.: Incentives for sharing in peer-to-peer networks. In: Proc. of the ACM conference on Electronic Commerce (2001)

51. Druschel, P., Rowstron, A.: Past: A large-scale, persistent peer-to-peer storage utility. In: HotOS VIII, pp 75-80. Schoss Elmau, Germany, May 2001

52. Pastry Project Team.: FreePastry API. Available at http://www.freepastry.org/

53. Yang, B., Garcia-Molina, H.: Ppay: micropayments for peer-to-peer systems. In: Proc. of the 10th ACM CCS. ACM Press (2003)

54. Catalano, D., Ruffo, G., Schifanella, R.: A p2p market place based on aggregate signatures. In: Proc. of ISPA Workshops, volume 3759 of LNCS, pp. 54-63. Springer, November 2005

55. Vishnumurthy, V., Chandrakumar, S., Sirer, E.: Karma: A secure economic framework for peer-topeer resource sharing. In: Proceedings of the Workshop on the Economics of Peer-to-Peer Systems. Berkeley, California, June 2003

56. Ntarmos, N., Triantafillou, P.: Seal: Managing accesses and data in peer-to-peer sharing networks. In: P2P '04: Proceedings of the Fourth International Conference on Peer-to-Peer Computing), pp 116123. IEEE Computer Society, Washington, DC, USA (2004).

57. Kamvar, S., Yang, B., Garcia-Molina, H.: Addressing the non-cooperation problem in competitive p2p systems. In: 1st Workshop on Economics of Peer-to-Peer Systems (P2PEcon'03), Berkeley, CA, June 2003

58. Wongrujira, K., Seneviratne, A.: Monetary Incentive with Reputation for Virtual Market-place based P2P. In: Proceedings of CoNEXT05. ACM Press, Toulouse, France, October 2005

59. Suryanarayana, G., Taylor, R.N.: A survey of trust management and resource discovery technologies in Peer-to-Peer applications. Technical Report UCI-ISR-04-6, Institute for Software Research, University of California, Irvine, July 2004

60. Jøsang, A., Ismail, R., Boyd, C.: A survey of trust and reputation systems for online service provision. Decision Support Systems (2006)

61. Marti, S., Garcia-Molina, H.: Taxonomy of trust: Categorizing p2p reputation systems. Computer Networks 50(4), 472-484 (2006)

\section{Author Biographies}

Giancarlo Ruffo Ph.D, is Associate Professor of Computer Science at the University of Torino, Italy, and active in Distributed Applications and P2P Systems. He is scientific supervisor of "W3Lab", cofounded with CSP, an Italian research center. He was involved in several research projects, publishing about 30 peer-reviewed papers in international journals and conferences. 
Rossano Schifanella Ph.D., is a researcher at the Department of Computer Science, University of Torino, Italy, where he is an active member of the Computer and Network Security Group. His research interests focus on distributed systems, Peer-to-Peer networks, digital marketplaces, social networking and decentralized recommender systems. 